\title{
REFLEXIONES SOBRE CUARENTA AÑOS DE EDUCACIÓN EN ESPAÑA O LA IRRESISTIBLE SEDUCCIÓN DE LAS LEYES
}

\author{
Reflections on forty years of education in Spain, or the irresistible \\ attraction of laws
}

\section{Manuel de Puelles Benítez*}

Fecha de recepción: 07/06/2015 • Fecha de aceptación: 15/06/2015

Resumen. En casi cuarenta años de democracia se ha originado un fenómeno que ha producido efectos contrarios a los buscados: una excesiva proliferación de leyes de educación ha traído una notable y constante inestabilidad legislativa. Se analizan aquí las razones profundas de este fenómeno, especialmente la política de los dos grandes partidos nacionales y su plasmación en las leyes de sus modelos sistémicos de educación, modelos sellados por la ideología respectiva, lo que inevitablemente ha conllevado un cambio legislativo cuando el electorado ha votado la alternancia democrática. Este análisis conduce a la necesidad de un nuevo consenso sobre educación que permita garantizar la aplicación efectiva de las reformas que las leyes impulsan.

Palabras clave: Socialdemocracia. Neoliberalismo. Neoconservadurismo. Políticas educativas. Consenso. Disenso.

Abstract. In almost forty years of democracy, educational policy in Spain has given rise to a phenomenon that has produced effects quite the opposite from those that were sought, with an excess of educational laws resulting in remarkable and constant legislative instability. This paper analyses the underlying reasons for this phenomenon, particularly the policies of the two major national parties and the embodiment in education laws of their systemic models of education, models which clearly bear the stamp of their respective ideologies. This has inevitably led to legislative reforms when the electorate has voted for a change of government. This analysis points to the need for a new consensus on education to ensure the effective implementation of the reforms launched by these laws.

Keywords: Social democracy. Neoliberalism. Neo-conservatism. Education policies. Consensus. Dissent.

* Universidad Nacional de Educación a Distancia. UNED. Facultad de Educación. Departamento de Historia de la Educación y Educación Comparada Juan del Rosal, 14. 28040 Madrid. España. mpuelles@edu.uned.es

Cómo citar este artículo: Puelles Benítez, Manuel de. «Reflexiones sobre cuarenta años de educación en España o la irresistible seducción de las leyes», Historia y Memoria de la Educación, 3 (2016): 15-44. 
Cuando en la sociedad coexisten unas creencias éticas extremadamente divergentes, el recurso a los derechos básicos no dará lugar a una solución que pueda ser aceptada como legítima. Si buscamos un compromiso legítimo y estable para cuestiones muy controvertidas, no tenemos más alternativa que la del largo camino de la política. [...] Este equilibrio no queda fijado para siempre, puede modificarse a medida que los intereses y las circunstancias cambian y que se conocen mejor los resultados de las diferentes políticas. Mientras que la adjudicación de los derechos aspira a la uniformidad y a lo definitivo, la práctica de la política permite llegar a soluciones cambiantes en diferentes circunstancias. ${ }^{1}$

En siglo y medio de historia sólo hemos tenido dos grandes leyes de educación. La primera fue el llamado Reglamento General de Instrucción Pública de 1821 que, aunque se aprobó con esa denominación por las Cortes del Trienio Constitucional, fue en realidad la primera ley general de educación que España tuvo, una ley que, a pesar de su brevísima duración-el fin del trienio liberal en 1823 trajo consigo su derogación-, sentó las bases del nuevo sistema educativo liberal y ejerció una extraordinaria influencia durante la primera mitad del siglo XIX. La segunda fue la Ley de Instrucción Pública de 1857, más conocida como ley Moyano por el ministro que la llevó a término, una ley que consagró el sistema educativo liberal y que, en contraposición a la de 1821, tuvo una presencia verdaderamente extraordinaria, pues su vigencia se extiende hasta 1970: lo que hoy llamamos la arquitectura escolar, esto es, la estructura de los diferentes niveles del sistema escolar y del sistema de enseñanza universitaria y superior permaneció prácticamente intacta hasta la promulgación de la ley de 1970.

Sin embargo, la Ley General de Educación de 1970 abrió un periodo caracterizado por la proliferación y superabundancia de leyes de educación. Concretamente, durante la democracia hemos visto aparecer siete leyes orgánicas que han incidido en la educación infantil, primaria, secundaria y formación profesional, a las que debe sumarse la reciente ley orgánica de Mejora de la Calidad de la Educación (LOMCE), publicada en diciembre de 2013 y convertida en la octava ley escolar; por otra parte, hay que contabilizar además tres leyes de reforma universitaria, sin olvidar que en la legislatura del Partido Popular (PP), transcurrida entre 2011 y 2015, se amagó con

\footnotetext{
${ }^{1}$ John Gray, Las dos caras del liberalismo (Barcelona: Paidós, 2001), 136.
} 
una nueva ley de reforma de la Universidad que factores probablemente internos aconsejaron aplazarla para otra posible legislatura. De haberse promulgado una nueva ley universitaria, habríamos llegado a tener doce leyes orgánicas de educación en un periodo que, desde el punto de vista histórico, podemos calificar de breve, esto es, el que se abre en 1975 con el tránsito de la dictadura de Franco a un régimen de democracia representativa y se cierra, por el momento, en 2015.

\section{¿PROLIFERACIÓN O PERVERSIÓN DE LAS LEYES?}

La situación actual arroja, pues, ocho leyes escolares y tres leyes universitarias. Si hacemos ahora un balance de las que han promulgado los dos grandes partidos que hasta ahora se han turnado en el Gobierno, habría prácticamente un empate técnico: seis leyes fueron obra del partido socialista y cinco lo han sido del partido conservador (incluyo aquí el Estatuto de Centros Escolares de la ya extinta Unión de Centro Democrático — UCD-, de 1980). No se trata, sin embargo, de establecer un paralelismo entre los dos grandes partidos, equidistancia a la que tan aficionada es en general la opinión publica no sólo porque estas leyes no tienen todas la misma entidad ni presentan la misma factura ideológica, sino porque el comportamiento político en ambos casos ha sido diferente: las leyes del Partido Socialista Obrero Español (PSOE) fueron votadas con el máximo consenso posible - prácticamente todo el arco parlamentario, salvo el PP que rehuyó el acuerdo y siempre votó en contra-, mientras que en las leyes del PP se produjo el fenómeno inverso, es decir, salieron adelante con el voto del partido que sostenía al Gobierno, gracias a la mayoría absoluta alcanzada en las urnas y, a veces, con el apoyo de minúsculos grupos parlamentarios, pero, habitualmente, con el voto en contra de todos los demás.

La sensación que ha provocado esta proliferación de leyes, no solo en la comunidad educativa sino, sobre todo, en la sociedad, es de preocupante inestabilidad. A este respecto es bueno recordar que en política es frecuente que las cosas no sean lo que son, sino lo que parece que son, lo que percibe el sujeto colectivo que las experimenta. ¿Qué percibe la sociedad? Percibe inestabilidad legislativa, y eso produce cansancio en la comunidad educativa, desmoralización en el profesorado, fatiga en los agentes sociales, y escepticismo general respecto de las reformas, aunque estas se presenten como el instrumento idóneo para mejorar la educación. 
Sin duda, once leyes orgánicas de educación son demasiadas para un periodo histórico tan corto. A este respecto, el PP suele decir que no ha habido tantos cambios, que en realidad dicho periodo lo han regido las leyes del partido socialista, que son las que han modificado a fondo el sistema educativo heredado del franquismo, especialmente la ley orgánica reguladora del Derecho a la Educación de 1985 (LODE), y otras dos leyes más, la ley orgánica de Ordenación General del Sistema Educativo de 1990 (LOGSE) y la ley orgánica de Educación de 2006 (LOE), que reformaron la ordenación y estructura del sistema estableciendo la arquitectura escolar del mismo. Esto es verdad pero sólo una parte de la verdad, ya que a esta afirmación hay que oponer dos matizaciones importantes: primera, que a las diferentes leyes, de uno u otro partido, han seguido en general cambios curriculares, algunos importantes, con la consiguiente perturbación para el profesorado y el alumnado; la segunda matización es que la ley orgánica de la Calidad de la Educación de 2002 (LOCE), primero, y la LOMCE de 2013, después, sí han incidido fuertemente en la estructura de la arquitectura escolar del sistema educativo.

Por tanto, sí ha habido excesivos cambios legislativos en tan breve periodo de tiempo. Es verdad que la proliferación legislativa es un fenómeno común a las democracias de nuestro tiempo, pero este fenómeno no se produce con la misma intensidad en todas las sociedades democráticas. En España, esta superabundancia de leyes se debe a razones intrínsecas, algunas de ellas perversas, que deben ser superadas.

El espectáculo servido por tantas leyes de educación está en contradicción con lo que desde Montesquieu llamamos el espíritu de las leyes. Conviene en este momento recordar algunos aspectos que, aunque elementales, a veces olvidamos. Las leyes, para alcanzar sus fines, necesitan conectar con la realidad. A las leyes no les basta con lograr la legitimidad política, necesitan también la legitimidad social, y ésta no se alcanza si no consiguen aportar soluciones a los problemas de la sociedad; por otra parte, la concepción democrática de las leyes nos enseña que nacen para regular la convivencia social y para dar seguridad a las relaciones entre los ciudadanos. De ahí su vocación de permanencia y de estabilidad. Sin embargo, lo que ha sucedido en estos últimos cuarenta años es que hemos visto nacer leyes que, al poco tiempo, daban paso a otras, normalmente de signo distinto, que producen muchas veces cambios de carácter pendular.

La aspiración de las leyes a la permanencia se ha visto menoscabada en nuestra democracia por la sucesión continua de normas reguladoras de as- 
pectos básicos de la educación. Es cierto que está en el espíritu de las leyes ser modificadas a lo largo del tiempo, bien para adaptarse a los cambios sociales, bien como consecuencia del progreso de la conciencia moral de la sociedad, pero la continua sucesión de leyes en materias importantes para la colectividad no sólo produce inseguridad, cansancio, relativismo y desencanto, sino que también traiciona el espíritu democrático de las misma leyes. Ello es particularmente cierto en lo que respecta a las reformas de la educación y a las leyes que deben garantizarlas.

Las leyes que regulan las reformas de la educación necesitan garantizar la estabilidad de esas reformas durante un largo plazo, necesitan en realidad sobrevivir al gobierno que las diseñó y las aprobó, necesitan de la continuidad en su aplicación. Necesitan del consenso. Un paso trascendental lo dio el pacto escolar del artículo 27 de la Constitución. Sin embargo, a ese pacto constituyente en educación le han seguido hasta el momento once leyes orgánicas, lo que nos obliga a formular la siguiente pregunta: ¿esta proliferación de leyes sobre una institución básica de la sociedad como es la educación no está revelando que el consenso constitucional del artículo 27 fue un requisito necesario pero no suficiente? Quede por el momento relegada esta pregunta mientras analizamos, primero, el presunto efecto de las leyes sobre el rendimiento del sistema escolar y, después, las verdaderas causas de esta proliferación.

\section{LEYES Y RENDIMIENTO ESCOLAR: ¿UNA RELACIÓN DE CAUSA- EFECTO?}

Desde que se puso en marcha la LOGSE ha sido frecuente en la literatura política del entorno del PP atribuir a esta ley la máxima responsabilidad sobre un presunto déficit de rendimiento escolar, o, cuando menos, ser la fuente de resultados mediocres, y desde luego causante de uno de los problemas reales del sistema educativo, el llamado fracaso escolar, consistente en que un número importante de alumnos no consigue obtener el título de graduado al término de la educación básica obligatoria (siendo por ello expulsados de manera inmediata del sistema escolar). Como es sabido, Ángel Gabilondo, ministro de Educación en la última legislatura socialista, presentó en abril de 2010 lo que llamó «Propuestas para un pacto social y político de la educación», avalado por más de un año de negociaciones con la comunidad escolar, las fuerzas sociales, los partidos políticos y las comunidades autóno- 
mas. Como es sabido también, el pacto no fue posible porque el PP rehusó firmarlo alegando que el Gobierno quería blindar un modelo que había fracasado por completo (la nota de prensa hablaba de «un rotundo fracaso»). Sobra decir que este modelo era el diseñado por la LOGSE, un modelo que, según esta fuerza política, había producido el fracaso escolar citado y un rendimiento del sistema cuando menos sumamente ineficiente.

Sobre el fracaso escolar los datos oficiales del Ministerio de Educación, publicados hasta el momento, ${ }^{2}$ nos dicen que, con el mismo modelo LOGSE, hay comunidades autónomas que registran una tasa bruta de fracaso muy por debajo de la media española, que en 2012 fue de un 24,9\%. A título de ejemplo, el País Vasco registró una tasa de 14,4\%, Asturias un 15,8\% y Cantabria un 17,5\%, mientras que otras comunidades sobrepasaron con mucho el promedio de España, tales como Murcia con un 29,3\%, Baleares un $32,6 \%$ y la Comunidad Valenciana un $37 \%$. Es decir, no parece que la arquitectura escolar de la LOGSE haya incidido sobre el problema del fracaso escolar, ya que hay comunidades que con ese modelo obtuvieron buenos logros en el camino del éxito escolar, mientras que otras siguieron soportando tasas de fracaso escolar por encima de la media nacional. Por otro lado, causa cierto desasosiego observar que entre la comunidad autónoma más exitosa (País Vasco) y la menos aventajada (Comunidad Valenciana) la diferencia entre ambas tasas de fracaso alcanza más de los veinte puntos porcentuales. Ello plantea otro problema, cual es el de la desigualdad territorial de la educación, esto es, que haya educandos que por nacer o vivir en una determinada comunidad tengan menos oportunidades educativas para lograr el éxito escolar que otros nacidos o con residencia en otra comunidad. La pregunta más imperiosa es responder a esta cuestión: ¿el ejercicio del derecho a la educación, garantizado en el artículo 27 de la Constitución, está condicionado en España por el lugar de residencia en una u otra comunidad? A la que puede sumarse otra no menos importante: ¿las leyes de educación influyen, o no, a la hora de obtener el éxito escolar?

Sobre la segunda cuestión planteada, el rendimiento del sistema escolar, el PP ha alegado también que la LOGSE ha sido responsable del bajo rendimiento en las evaluaciones PISA que se realizan cada tres años. Permítaseme una observación previa: la interpretación que suele darse a los informes

\footnotetext{
${ }^{2}$ Instituto Nacional de Evaluación Educativa, Sistema estatal de indicadores de la educación (Madrid: Ministerio de Educación, Cultura y Deporte, 2014), 93.
} 
PISA es en general muy deficiente. Se acostumbra decir que, según estos informes, España no obtiene buenos resultados en la evaluación del rendimiento del sistema escolar. A este respecto es curioso el efecto hipnótico que los informes PISA están produciendo en el mundo de la educación, no sólo en España sino también en el continente europeo o americano:

el poder de los rankings generados a partir de los datos de PISA es tal que su mera publicación se convierte en el detonante de enconadas discusiones políticas a escala nacional. [...] esta presencia [de PISA] es superficial y amplifica todo cuanto arroja una imagen negativa del sistema escolar del país. En efecto, la presencia mediática contribuye enormemente a generar la imagen pública del sistema escolar de un país en la opinión pública y, generalmente, su efecto es devastador. ${ }^{3}$

Sin embargo, quiero dejar constancia de que, contrariamente a lo que los medios de comunicación suelen difundir y el PP suele alegar de consuno, España está muy cerca de la media de PISA, no sólo en el último informe, el de 2012, sino también en los anteriores.Tomando como ejemplo la referencia a los rendimientos en la competencia matemática (aunque en lectura y ciencias los resultados son similares), comunidades como Castilla y León o Asturias, gobernadas por el PP y el PSOE desde hace varios o muchos años, han conseguido en el informe PISA 2012, publicado en 2013, resultados académicos por encima de la media de los países de la OCDE. También lo han conseguido otras comunidades gobernadas por otros partidos distintos de los citados como Navarra o País Vasco, sin olvidar que otras comunidades están muy cerca de la media OCDE y por encima del promedio de España; ${ }^{4}$ no está de más recordar que comunidades autónomas gobernadas por los dos partidos turnantes, obtienen rendimientos por debajo de las medias nacionales o internacionales, como es el caso, por ejemplo de Islas Baleares o Andalucía. Obvio es decir que todas estas comunidades, hayan o no superado dichos promedios, tanto en PISA 2012 como en otros anteriores, gobernadas por uno u otro partido, utilizaron el modelo de enseñanza regulado por la LOGSE. No parece, pues, que sea el modelo el que

\footnotetext{
${ }^{3}$ Francesc Pedró, «Deconstruyendo los puentes de PISA: del análisis de resultados a la prescripción política», Revista Española de Educación Comparada, 19 (2012): 142.

${ }^{4}$ Instituto Nacional de Evaluación Educativa, Sistema estatal, 73.
} 
esté condicionando lo que convencionalmente se considera un rendimiento escolar mejor o peor. Tampoco puede imputarse al partido que ha gestionado la educación en las diferentes comunidades autónomas, sino otros factores en los que ahora no podemos entrar (entre ellos podemos destacar la educación de los padres, especialmente lo que los especialistas denominan el «diferencia sexual», esto es, la educación de la madre, la clase social a la que pertenece el alumno, la acumulación cultural de las comunidades producida por una tradición histórica, etc.).

Por otra parte, volviendo a PISA 2012, estamos en competencia matemática a 10 puntos de la media de los países de la OCDE, a 8 en comprensión lectora y a 5 en la competencia científica. Los sociólogos de la educación, especializados en estos temas, nos dicen que dado el margen de variación y error de tipo estadístico que hay siempre en las evaluaciones muestrales -PISA no hace evaluaciones censales-, las diferencias de cinco o diez puntos respecto de 500 que es la media OCDE no son significativas desde el punto de vista del rendimiento de un sistema. Más aún, los mismos especialistas de la OCDE nos dicen que, exceptuada la mayoría de los países asociados que están en mala posición (especialmente América Latina y un buen número de países de Asia de los que apenas se habla), exceptuados los países asociados, repito, la mayor parte de los países miembros se encuentran en la franja central, en la que también está España. Finalmente, apenas se ha señalado que España, en Pisa 2012, se encuentra justamente en el promedio de la Unión Europea: sólo a 5 puntos en matemáticas, a un punto en lectura y a otro en ciencia. Es decir, que estamos en el nivel medio europeo, lo que debemos destacar si tenemos en cuenta la situación de la que se partía en 1970 y el camino recorrido en un periodo que considerado históricamente es muy reducido. Por supuesto, esto no quita para recordar también que no estamos entre los siete u ocho países que tienen los mejores sistemas educativos. Queda, pues, un largo camino que recorrer. Y la proliferación legislativa, es, como veremos, un obstáculo importante a superar.

Dicho esto, debemos hacernos ahora otra pregunta importante: dado el agotador esfuerzo que los ministerios de Educación de los diferentes gobiernos han dedicado y dedican a la confección de una ley tras otra -once leyes, no lo olvidemos-, ¿no habría sido mejor aplicar ese esfuerzo a la elaboración de políticas dirigidas a mejorar la educación, políticas que puedan sostenerse a medio y largo plazo y que por tanto puedan consolidar las 
mejoras alcanzadas y subsanar las deficiencias detectadas? La elaboración de políticas no exige por lo general el concurso de las leyes, tanto más si esas políticas se basan en un pacto previo de sostenibilidad. Para ello nuestra clase política tendría que vencer la irresistible seducción que las leyes le producen, superar la tentación de que la victoria electoral tenga necesariamente que plasmarse en las páginas del Boletín Oficial del Estado, acabar con la concepción taumatúrgica de las leyes, tantas veces desmentida por la realidad. Para todo ello, para llegar a puerto tenemos, no sólo la clase política, que reflexionar sobre las enormes dificultades que se oponen a otra vía alternativa, la de sustituir las leyes por políticas sostenidas. Para ello hay que hacerse otra pregunta: ¿por qué se ha producido esta situación? Yo creo que hay una razón profunda detrás de todo ello. Estamos ante una situación que pone de relieve la existencia de un conflicto de carácter permanente que exige una explicación y una interpretación. Es lo que voy a intentar ofrecer a continuación.

\section{LAS RAZONES PROFUNDAS DE UN CONFLICTO ENDÉMICO}

Las dificultades citadas existen porque la educación ha conservado desde la irrupción de la modernidad política un doble carácter: una vertiente pública, derivada del principio de igualdad, que implica el acceso de todos los ciudadanos al derecho a la educación, y una vertiente privada, que pone el acento en la libertad de crear y elegir centro docente. Esta doble vertiente, esta consideración de la educación como un derecho complejo y bifronte - de un lado, es un bien privado que afecta al desarrollo de la personalidad del individuo, pero, de otro, es un bien público con extraordinarias repercusiones sociales, económicas, políticas y culturales- explica la existencia de una larga y conflictiva historia escolar en la que los principios de igualdad y libertad, aplicados a la educación, se enfrentaron a lo largo de dos siglos de historia española, originando un conflicto que se ha alimentado de fuertes pasiones políticas y religiosas.

Esta ambivalencia, producto de concepciones diversas, se produjo también en otros campos, pero la educación fue en el pasado, y sigue siéndolo hoy, un terreno propicio para el enfrentamiento de discursos ideológicos que, muchas veces, se han presentado, y se presentan, como contrarios, incompatibles y, por tanto, mutuamente excluyentes. En el marco de este enfrentamiento, lo que en realidad se está cuestionando es el rol del Estado 
en la educación. Si se considera la educación como un derecho predominantemente privado, el Estado no pasa de ser un actor puramente subsidiario, y si se parte de la consideración de la educación como un derecho predominantemente público, el Estado se convierte en garante del servicio público de la educación, lo que exige una decidida intervención de los poderes públicos.

Esta doble tradición histórica la siguen encarnando en la actualidad el PP y el PSOE, respectivamente. Es verdad que hubo un pacto escolar, el del artículo 27 de la Constitución que consistió esencialmente en conciliar el principio de igualdad, reconociendo el derecho de todos a la educación, y el de libertad, reconociendo la libertad de enseñar y de elegir centro docente. Pero, por razones en las que ahora no podemos entrar, el pacto escolar del artículo 27 ha ido perdiendo virtualidad a lo largo de todos estos años. Durante la primera etapa de gobierno socialista (1982-1996) pareció que el pacto persistía, si bien con notables disfunciones, pero la llegada al gobierno del partido conservador en 1996, refundida Alianza Popular en el Partido Popular, mostró que los dos modelos seguían siendo divergentes y que la alternativa de gobierno significaba de nuevo una oscilación pendular sin que el pacto del artículo 27 sirviera de protección para mitigar esa divergencia. Desde el principio de la nueva legislatura del partido conservador (1996-2000).

se plantearon con toda la vehemencia imaginable los debates entre escuela pública y privada, la enseñanza de la religión y sus alternativas, los contenidos de los programas [curriculares], el protagonismo generalizado de las comunidades autónomas, etc. Se reabrieron «heridas» a raíz de nuevas batallas entre dos visiones antagónicas de la pedagogía y los principios educativos. ${ }^{5}$

Como veremos, la derecha actual, neoliberal y neoconservadora, ha conseguido contaminar todo lo que toca, quebrantando incluso uno de los principios que están en la base de todas las democracias occidentales, el ideal de ciudadanía. Este ideal, que brota de la igualdad, sigue siendo uno de los principios básicos que han dado a luz al Estado democrático; su esencia se caracteriza en que la soberanía reside en el cuerpo políti-

\footnotetext{
${ }^{5}$ Francisco Michavila, «Política educativa», en El Gobierno de Aznar. Balance de una gestión, 1996-2000, ed. Javier Tusell (Barcelona: Crítica, 2000), 125.
} 
co, integrado por todos los ciudadanos. Como afirmó Robert Moore en el prefacio a la nueva edición en español de las famosas conferencias que el sociólogo T. H. Marshall dictó en 1949 en la Universidad de Cambridge, la nueva derecha, surgida en la década de los ochenta del pasado siglo, tuvo desde sus inicios como objetivo fundamental su pugna con la idea de la ciudadanía:

la idea de ciudadanía es uno de tantos dislates progresistas que sólo sirven para calentar los cascos de la gente respecto al espacio social que ocupa, y para que los súbditos acaben por creer que no son sólo eso, súbditos obligados a dejarse gobernar sino también personas dotadas de derechos. ${ }^{6}$

Se piensa así, sin duda, porque la ciudadanía conforma derechos que interfieren la mecánica del mercado. Por otra parte, la izquierda ha tardado en asimilar algunos de los derechos que conforman la libertad de enseñanza, especialmente el de crear y elegir centro docente, considerados muchas veces como una amenaza para la educación pública.

De esta forma, podríamos decir que la tentación de la derecha española consiste en relegar al reino de las sombras los contenidos básicos de la igualdad y centrarse sólo en el principio de libertad, sucumbiendo así a los cantos de sirena del discurso neoliberal en educación; por el contrario, la tentación de la izquierda española se traduce en eludir las políticas integradoras que lleven consigo aplicar la libertad y la igualdad para todos, haciendo énfasis no sólo en la igualdad sino también en la necesidad —y en el derecho- de educar en la libertad y para la libertad, derechos todos, tanto en un caso como en el otro, reconocidos y garantizados por la Constitución española.

Veamos, pues, cómo se ha ido produciendo este conflicto endémico en las políticas legislativas de los dos grandes partidos nacionales (a la hora de redactar estas reflexiones nadie sabe aún cómo y cuánto puede alterar este cuadro la irrupción en el mapa político de otras fuerzas nuevas que aspiran a la regeneración política y al cambio, tanto a la izquierda como a la derecha de la arena política).

\footnotetext{
${ }^{6}$ Rober Moore, «Prefacio», en Ciudadanía y clase social, T. H. Marshall y Tom Bottomore (Madrid: Alianza, 1992), 9.
} 


\section{DEL SOCIALISMO A LA SOCIALDEMOCRACIA: LAS POLÍTICAS DE IGUALDAD DE ENSEÑANZA}

En los últimos años del tardofranquismo el PSOE se había situado más allá de la izquierda del espectro político, adoptando un discurso que «sintonizaba con ese espíritu de cambio radical que en Europa había simbolizado, mejor que cualquier otro acontecimiento, la revuelta de mayo de $1968 » .^{7} \mathrm{~A}$ ello hay que sumar la influencia que tuvo en el PSOE la renovación del socialismo francés en 1971 bajo la dirección de Mitterand y la experiencia chilena que por esos años intentaba implantar el socialismo por cauces democráticos. En el XIII Congreso del partido en el exilio, celebrado en Suresnes en octubre de 1974, la joven generación de socialistas del interior triunfó sobre la vieja e histórica dirección que había sobrevivido a la Guerra Civil, consiguiendo imponer un programa radical y revolucionario que incluía el derecho a la autodeterminación de los diferentes pueblos de España y la definición del PSOE como un partido marxista y revolucionario que aspiraba a la toma del poder político y económico por la clase obrera, transformando la sociedad capitalista en una sociedad socialista. Ello no excluía, sin embargo, una estrategia dirigida a recobrar las libertades si bien desde una perspectiva de clase, «libertades que se valoraban fundamentalmente por su utilidad en cuanto a facilitar el logro de una democracia socialista. En este sentido, se afirmaba, la "ruptura democrática" sería revolucionaria». ${ }^{8}$ Este programa iba también acompañado de la aceptación de la democracia y de sus valores, lo que apuntaba ya a contradicciones internas que no tardarían mucho en aflorar:

Para ellos [los jóvenes socialistas] el socialismo se definió muy pronto como una conquista de la libertad frente a la dictadura y como una lucha por la sociedad socialista contra el capitalismo, lo que llevaba a incorporar a su lenguaje político, por una parte, los valores propios de la democracia y, por otra, potencialmente contradictoria, a reafirmar el «carácter revolucionario» del socialismo. ${ }^{9}$

Todo ello explica que en los primeros momentos del posfranquismo lo que predominó en el PSOE fue la radicalidad ideológica de sus principios,

\footnotetext{
${ }^{7}$ Juan Avilés Farré, «El proyecto socialista: del marxismo a la modernización», en Historia de la época socialista. España: 1982-1996, dirs. Álvaro Soto Carmona y Abdón Mateos López (Madrid: Sílex, 2013), 14.

${ }^{8}$ Richard Gillespie, Historia del Partido Socialista Obrero Español (Madrid: Alianza, 1991), 317.

${ }^{9}$ Santos Juliá, Los socialistas en la política española, 1979-1982 (Madrid: Taurus, 1997), 398.
} 
fenómeno cuya significación exige analizar, aunque sea brevemente, el contexto histórico en que se desarrolló.

La muerte de Franco en noviembre de 1975 puso fin a un régimen político cuya naturaleza, precisamente por su larga duración, presenta aún cierta complejidad. Ciertamente, no es el momento de ocuparse de este problema, pero sí de destacar que hubo, al menos, dos rasgos que permanecieron constantes a lo largo de casi cuarenta años: uno de ellos fue que, desde el principio hasta el final, el franquismo se caracterizó por ser un régimen de poder personal, una dictadura; la segunda característica que nos interesa subrayar es que fue un régimen fruto de una cruel guerra civil y, en consecuencia, de la imposición violenta de unos españoles sobre otros. Estas brevísimas consideraciones contextuales son importantes porque explican en alto grado una de las particularidades del periodo preconstituyente en que se desarrolló la Transición, esto es, la existencia de una sobrecarga ideológica que se manifestó especialmente en la oposición clandestina al régimen, de la que tuvieron que despojarse las fuerzas políticas para poder dar paso pacíficamente a un cambio de régimen con vocación de permanencia. Aunque el PSOE será pronto uno de los actores principales, sólo lo fue a costa de echar por la borda la sobrecarga de su radicalidad.

No obstante, todavía en el XXVII Congreso celebrado en España, en diciembre de 1976 en condiciones de semiclandestinidad, el PSOE se definió como un partido que aspiraba a «superar el modo de producción capitalista mediante la toma del poder político y económico y la socialización por la clase trabajadora de los medios de producción, distribución y cambio», ${ }^{10}$ debatiéndose incluso la oportunidad de una alianza entre socialistas y comunistas para formar un bloque anticapitalista de clase. En educación, el XXVII Congreso, en una nueva etapa que se consideraba de transición al socialismo, puso el acento sobre la planificación y la gestión democráticas de la enseñanza de acuerdo con los criterios siguientes: escuela pública única, lo que significaba la progresiva desaparición de la enseñanza privada; enseñanza gratuita; enseñanza laica; establecimiento de un ciclo único —desde la educación básica hasta el bachillerato y la formación profesional—; cuerpo único de profesores e implantación del servicio público de la educación, considerado en sentido estricto, es decir, como un servicio único y excluyen-

10 Antonio López Pina, La generación del 56 (Madrid: Marcial Pons, 2010), 331. 
te. Así pues, en el contexto político del posfranquismo el PSOE se sitúa muy a la izquierda del nuevo arco político.

En los primeros meses del posfranquismo se pudo observar la aparición de dos grandes bloques muy definidos: uno, conservador, partidario de reformas políticas de diversa entidad, pero siempre dentro de la legalidad vigente, integrado por Alianza Popular (AP) y Unión de Centro Democrático (UCD); otro, representado por la oposición histórica al franquismo, partidario de la ruptura política y de la implantación ex novo de un régimen democrático, formado por el Partido Comunista de España (PCE) y el PSOE. En el primer bloque, AP, en tanto que heredero directo del franquismo, se situará más a la derecha, mientras que UCD buscará desde el principio, como su propio nombre fundacional preanunciaba, el centro político; en el segundo bloque, el PSOE se colocará a la izquierda del PCE, aunque en poco tiempo sufrirá una acelerada evolución hacia el centro, mientras que el PCE, muy castigado en su larga etapa clandestina, se adaptará mejor a la nueva circunstancia de la transición política, aportando moderación. Todas estas posiciones tendrán su repercusión en el tratamiento de los problemas que el franquismo había dejado pendientes en educación que, como he indicado en otro lugar, eran fundamentalmente tres: «el problema de la escolarización o de la extensión de la enseñanza, el problema de la calidad de la educación y el problema de la democratización de las instituciones educativas». ${ }^{11}$ Resumiendo, puede decirse que el PSOE concedía primacía absoluta al principio del derecho a la educación, si bien en el bienio 19771978 va a sufrir un giro espectacular en sus posiciones que le conducirá al consenso constitucional y, por ello, a posiciones más moderadas y flexibles.

Aunque en la primavera de 1979, en el XXVIII Congreso del partido, triunfó la ponencia que ratificaba la condición del PSOE como partido marxista, Felipe González se declaró incompatible con ese programa y presentó su dimisión como secretario general. En el fondo, lo que Felipe González estaba denunciando en ese congreso era la acumulación ideológica del partido, que abría una enorme distancia entre el viejo programa máximo — «un resto arqueológico de las visiones primitivas e ingenuas de la II Internacional»- y las necesidades de «una sociedad que había sufrido grandes transformaciones económicas, sociales y culturales durante los últimos años del

${ }_{11}$ Manuel de Puelles Benítez, Educación e ideología en la España contemporánea (Madrid: Tecnos, 2010), 367 y ss. 
franquismo». ${ }^{12}$ Se produjo entonces una crisis profunda que desembocó en la creación de una Comisión gestora encargada de preparar un congreso extraordinario. Este congreso, reunido en septiembre de ese mismo año, dio un vuelco a las posiciones más radicales: el PSOE dejó de ser un partido marxista y socialista para convertirse en un partido socialdemócrata, homologable con los demás partidos europeos de este signo, lo que le abrió el camino hacia el triunfo electoral de 1982.

En el primer gobierno socialista se practicó un discurso de moderación inserto en la línea socialdemócrata europea de aceptación del mercado y de aplicación de políticas sociales de redistribución, entre las que ocupaba un lugar importante la política educativa. En dicho gobierno, José María Maravall se hizo cargo de la cartera de educación, promoviendo una política de signo socialdemócrata y diseñando las estrategias igualitarias cuya realización posterior, sin embargo, no se mantuvo con la misma firmeza por los ministros que le sucedieron. En este diseño la ley orgánica del Derecho a la Educación (LODE) de 1985 ocupó un lugar central, no sólo porque reguló el acceso a este derecho, sino también porque ordenó las relaciones de la enseñanza privada con el servicio público de la educación, estableciendo el modelo de conciertos, esto es, garantizando la financiación de la educación básica obligatoria en las escuelas privadas siempre que coadyuvaran, dice la ley, al servicio público de la educación. Como se ha señalado, cabría decir

que el logro más radical de los socialistas, al menos en potencia, fue la reforma hecha por Maravall de la enseñanza primaria y la secundaria. La nueva ley, a la que se opusieron movilizaciones de masas organizadas por la Iglesia y por la derecha, que temían perder influencia como resultado de ella, hizo mucho por la democratización de la administración de las escuelas, al hacer que el cambio en ellas constituyera una condición previa de la financiación pública. ${ }^{13}$

Como es sabido, no hace mucho, en 2010, se celebró el 25 aniversario de la LODE. A primera vista, y dado que la mayoría de las leyes escolares han sido derogadas, parecería que estamos ante un éxito de estabilidad legislativa. Ello es verdad sólo en una pequeña parte: se ha consolidado el modelo de concierto pero al precio de pervertir el espíritu originario de la ley.

\footnotetext{
12 Javier Pradera, Corrupción política. Los costes de la democracia (Barcelona: Galaxia Gutenberg, 2014), 64.

13 Gillespie, Historia del Partido, 434. La cursiva es mía.
} 
Como botón de muestra, recuérdese que, gracias a diferentes estrategias, más o menos toleradas, los centros concertados no son en realidad gratuitos; el concierto es hoy más bien una subvención de ayuda al precio (lo que equivale a decir que la LODE no desplegó toda su potencialidad). Por otra parte, la LODE no ha permanecido intacta: la modificó el PP en su primera etapa de gobierno, la volvió a modificar el PSOE en su segunda etapa a fin de retrotraer la ley a su espíritu y letra originales, y la ha vuelto a modificar ahora el PP con la LOMCE, desvirtuándola de nuevo. Es decir, la LODE no es, en mi opinión, un ejemplo de estabilidad legislativa, sino más bien todo lo contrario. Tampoco la LOE va a serlo, modificada profundamente por la LOMCE, a pesar de que durante sus cinco años de plena vigencia demostró su operatividad al servicio de la igualdad de oportunidades.

En el segundo periodo del gobierno socialista (2004-2011) cabe distinguir dos partes muy diferenciadas. En la primera legislatura, Rodríguez Zapatero hizo en su discurso de investidura un fuerte hincapié en el pleno desarrollo de los derechos de ciudadanía dando lugar a lo que, en terminología acuñada por Pettit —el gran politólogo del republicanismo moderno-, se denominó una política civicista. Como ha señalado una de las cabezas pensantes del socialismo español, la primera legislatura de Rodríguez Zapatero se caracterizó por una doble exigencia, recuperando «un radicalismo liberal, de carácter político y social, y un radicalismo democrático. Esto representa[ba] una recuperación de cuestiones referidas a libertades y derechos políticos que la socialdemocracia había dejado un tanto de lado». ${ }^{14}$ La singularidad de este giro estuvo en que las políticas civicistas no significaron una renuncia a los postulados clásicos de la socialdemocracia, porque el giro político apuntó no solo a la ampliación de los derechos civiles, sino también de los derechos sociales. De este modo, junto a leyes de inspiración netamente republicana como la ley del matrimonio homosexual, la de protección de la mujer frente a la violencia de género, el «divorcio exprés», la ley de plazos del aborto o la de igualdad de derechos entre hombres y mujeres, se promulgaron leyes de ampliación de los derechos sociales como la ley de dependencia o la nueva ley orgánica de Educación de 2006 (LOE).

No se ha subrayado lo suficiente, a mi juicio, que la LOE fue una ley que, además de subsanar errores cometidos en la aplicación de la LOGSE,

14 José María Maravall, «Epílogo. La socialdemocracia de Zapatero», en La España de Zapatero. Años de cambio 2004-2008, Anna Bosco e Ignacio Sánchez-Cuenca (Madrid: Pablo Iglesias, 2009), 274. 
apuntó a una meta socialdemócrata de igualdad, buscando conciliar la máxima calidad de la educación con la máxima equidad posible. La ley sentó las bases para la realización de políticas dirigidas a hacer posible la igualdad de oportunidades: políticas de refuerzo del aprendizaje para alumnos con problemas de este tipo, diversificación curricular y desdoblamiento de aulas en materia fundamentales (como lengua y matemáticas) para alumnos con problemas para obtener el título de Graduado en la educación básica obligatoria, evaluaciones de diagnóstico para detectar de modo temprano los problemas de aprendizaje, potenciación de la orientación escolar y de la tutoría (mención especial merece el programa PROA - Programa de Refuerzo, Orientación y Apoyo-, de extraordinario éxito, defendido incluso por las comunidades gobernadas por el PP y que fue totalmente desmantelado por los presupuestos generales del Estado de 2012). Otra importante preocupación de la LOE desde el punto de vista de la igualdad fue el tratamiento de un problema del que en 2006 apenas se hablaba, el de la desigualdad territorial en educación; esto es, el hecho de que hubiera diferentes oportunidades según se naciera o viviera en una determinada comunidad autónoma o en otra. El instrumento escogido fue la realización de políticas de cooperación territorial entre el Estado y las comunidades, dirigidas a reducir esas desigualdades, política que el ministro Gabilondo dotó de amplios recursos financieros y que llevó a cabo con éxito. Sin embargo, como es sabido, con la LOMCE, la LOE ha sido objeto de una modificación sustancial que afecta notablemente al principio de igualdad de oportunidades en la educación.

La segunda legislatura de Rodríguez Zapatero estuvo marcada por dos circunstancias importantes. Una de ellas fue producto de la implantación de la LOE; la otra, de carácter prácticamente mundial, fue obra de la Gran Recesión que se manifestó con toda su crudeza en 2008.

Según los últimos datos oficiales que tenemos hasta el momento, ${ }^{15}$ hubo durante la segunda legislatura un hecho que no ha sido subrayado suficientemente y fue una importante reducción del llamado fracaso escolar, esto es, del porcentaje de alumnos que no conseguían el título de graduado al culminar la Educación Secundaria Obligatoria (ESO): en 2007, año en que empezó a aplicarse la LOE, el porcentaje fue de un 30,6\%, mientras que en 2011, cuando terminó la legislatura, fue de $24,9 \%$, lo que significó un descenso en ese periodo de casi seis puntos porcentuales, o, lo que es lo mismo,

15 Instituto Nacional de Evaluación Educativa, Sistema estatal, 93. 
miles de alumnos consiguieron el título y pudieron continuar sus estudios en la educación postobligatoria. Esta importante disminución de la tasa del fracaso escolar repercutió lógicamente en la reducción que por esos años se produjo también en el abandono escolar temprano, aunque aquí probablemente influyera más el hundimiento del sector de la construcción, con la consiguiente vuelta de muchos alumnos a las aulas. En todo caso, parece claro que algunas medidas de la LOE, solicitadas de modo apremiante por la comunidad escolar, contribuyeron a la mejora de estos resultados.

La otra circunstancia fue muy negativa para el Gobierno socialista, aunque en general también lo fuera para los gobiernos europeos, sometidos todos ellos a la dura prueba de una crisis económica como no se había visto desde 1929; desbordados por la intensidad y gravedad de la crisis, pagaron su precio con la derrota en las urnas. La Gran Recesión de 2008 colocó a Rodríguez Zapatero ante un dramático dilema: o políticas de recortes o políticas sociales. Aunque el presidente dejó a salvo la educación y la sanidad públicas, las consecuencias de los recortes efectuados fueron devastadoras para su gobierno, que sufrió una considerable derrota en las elecciones generales de finales de 2011. El PP obtuvo el mayor triunfo electoral de su historia. A finales de ese año alcanzaba el poder tanto en los grandes municipios como en las comunidades autónomas y en el gobierno de la nación.

\section{DEL POSFRANQUISMO AL NEOLIBERALISMO: LAS POLÍTICAS DE LIBERTAD DE ENSEÑANZA Y LAS TENSIONES CON EL NEOCONSERVADURISMO}

En 1977 España se encontraba en una fase de la Transición que un politólogo podría calificar de pugna entre concepciones opuestas, pugna que se extendía a todas los aspectos de la vida pública. ¿Cuál era, entonces, el programa de las principales fuerzas políticas sobre educación? Entre los documentos de aquel tiempo - fuentes primarias para nosotros- destaca por su riqueza de contenidos la trascripción que una revista especializada realizó de la mesa redonda convocada para conocer las propuestas de los partidos políticos, en un momento en que la elaboración del texto constitucional estaba próxima. La Revista de Educación requirió a los partidos políticos que habían obtenido representación parlamentaria en las Cortes recién inauguradas a fin de que, en un momento en que aún se desconocían los trabajos de la Ponencia constitucional, dieran a conocer su opinión «sobre las cuestiones referentes a 
la educación que deberían ser incorporadas al texto constitucional en elaboración». ${ }^{16}$ Salvo el PSOE, todos los partidos acudieron a la cita.

Alianza Popular (AP), partido constituido en los comienzos de la Transición como refugio del franquismo residual, defendió como «primer principio» de su programa la defensa de la libertad de enseñanza, entendida fundamentalmente como «libertad de creación de centros por personas, grupos y por el propio Estado», incluyendo en ella la «libertad discente o de elegir centro de enseñanza», mientras que el derecho a la educación — «el derecho y el deber de educarse»— fue en su programa el «segundo principio». El soporte de la libertad de enseñanza lo basó en la consideración de «la educación como servicio a la sociedad (y no como servicio público con sus connotaciones administrativas)»; $;{ }^{17}$ en consecuencia, la libertad de enseñanza debía ser subvencionada (de ahí que AP hablara del servicio social de la educación). De este modo, el círculo ideológico se cierra: las subvenciones aparecen como el soporte real de la libertad de enseñanza, sin que el programa de AP previera contraprestación alguna por parte de los receptores de la subvención.

La Unión de Centro Democrático (UCD), partido creado desde el Gobierno para acudir a las primeras elecciones generales, las de 1977, representaba la derecha más moderna, aspirante a ocupar el centro. Hacía también un especial hincapié en la libertad de enseñanza en el sentido ya indicado, pero polarizado sobre todo hacia lo que en aquel momento se llamaba «pluralismo ideológico o social» de los centros docentes (poco tiempo después identificado como el ideario de los centros); en consecuencia, defendía que

las distintas instituciones puedan articular sus propios proyectos educativos en centros docentes concretos y que sería contradictorio con el principio de libertad y con el principio de pluralismo social el exigir un pluralismo interno de los centros que surgen precisamente para facilitar opciones diferenciadas. ${ }^{18}$

En realidad, lo que había detrás de este «pluralismo social» de los centros era una defensa firme de las escuelas privadas confesionales. Se defendía también, en este caso por UCD, la subvención como sostén ma-

\footnotetext{
16 «Mesa redonda con los partidos políticos con representación parlamentaria en torno a la educación en el proyecto constitucional», Revista de Educación, 273 (1977): 153.

17 «Mesa redonda», Revista de Educación, 273 (1977): 155.

18 «Mesa redonda», Revista de Educación, 273 (1977): 163.
} 
terial de la libertad de enseñanza pero, tratándose de fondos públicos, se consideraba adecuado que el Estado estableciera determinadas condiciones para su otorgamiento. Asimismo, los centristas se diferenciaban de AP porque defendían el principio de igualdad de oportunidades y, por tanto, la gratuidad de la educación básica, si bien, alegando razones financieras, se postulaba una aplicación gradual.

Como es sabido, tras el hundimiento de la UCD en 1982, AP se benefició ampliamente de dicha situación pasando a constituir el principal partido de la oposición. Sin embargo, las sucesivas victorias del PSOE evidenciaron que AP tenía un techo electoral que no podía superar. Ello explica que a finales de los años ochenta se produjera un importante giro político. Así, en 1989 se produjo en la derecha española un hecho significativo, entró en la arena política

una nueva generación cuyas referencias culturales eran principalmente anglosajonas - y quizá más británicas que norteamericanas, lo que explicaría la devoción por Margaret Thatcher-; estos nuevos políticos, además, habían visto derrumbarse algunos de los mitos importantes para la generación anterior. El año de 1989 fue la fecha de la renovación de la antigua Alianza Popular pero también de la caída del comunismo. ${ }^{19}$

De este modo, entraba en la lid política una nueva generación que no había hecho la Transición y que pugnaba por encontrar un modelo para la derecha española que no tuviera como núcleo central el franquismo residual. Lo encontró en la nueva derecha británica —English New Right-, la que capitaneó Margaret Thatcher en los años setenta llevando al partido conservador al poder en 1979. El "giro al centro» del PP fue en realidad la adopción del thatcherismo a la situación española. El thatcherismo, sin embargo, no fue un modelo unívoco. No lo fue porque, como he señalado en otro lugar, en él convergían «dos tendencias distintas: el neoliberalismo, que propugna la libertad de los mercados a toda costa, y el neoconservadurismo, que se enfrenta a los problemas actuales apelando a la tradición y los viejos valores». ${ }^{20}$

\footnotetext{
19 Javier Tusell, El aznarato. El Gobierno del Partido Popular 1996-2003 (Madrid: Aguilar, 2004), 56.

${ }^{20}$ Manuel de Puelles Benítez, «La influencia de la Nueva Derecha inglesa en la política educativa española (1996-2004)», Historia de la Educación. Revista interuniversitaria, 24 (2005): 234.
} 
El thatcherismo fue una combinación de dos ideologías no totalmente compatibles: el neoliberalismo — partidario de un radical laissez faire- y el neoconservadurismo - defensor de un individualismo no menos radical- . El primero hundía sus raíces en Adam Smith y su máximo exponente era Friedrich Hayek, opuesto a la planificación económica porque la conducta humana no puede «abarcar la infinita variedad de las diversas necesidades de las diferentes personas que compiten por los recursos disponibles y asignar un peso definido a cada una», mientras que el libre mercado se presenta como un sistema desarrollado espontáneamente por muchas generaciones. ${ }^{21}$ Es decir, frente a lo que en aquellos momentos era el dogma predominante de la planificación económica, Hayek contraponía el dogma de un mercado caracterizado por la libre competencia y por la autorregulación: para el padre del neoliberalismo el libre mercado se aseguraba con un mínimo de Estado (ejército y policía fundamentalmente a fin de preservar el orden público que los mercados necesitaban). Al final todo se basaba en una concepción optimista de la vida económica: aunque los hombres persigan su propio bien, el mercado transformará ese bien privado en un bien público. Los neoconservadores, en cambio, partían de una concepción pesimista del hombre, claramente hobbesiana: es necesario un Estado fuerte para controlar el mal (y a veces para proteger al débil). Las buenas costumbres, los viejos valores tradicionales —en definitiva, los valores victorianos-, el orden, todo ello son clave en la concepción neoconservadora del Estado y de la política. Estamos en realidad ante una reacción frente a la sociedad permisiva de los años sesenta del pasado siglo, a la que se culpaba de los bajos estándares existentes en las escuelas.

El thatcherismo albergaba, pues, a dos sectores ideológicos diferentes: el ala neoliberal representaba la primacía del mercado frente al Estado, lo que significaba menos regulación estatal y menos impuestos; la fracción neoconservadora representaba una coalición de intereses que demandaba una fuerte reacción frente a los problemas económicos, sociales y culturales, es decir, una vuelta a los viejos valores de la tradición que instituciones como el Estado y la Iglesia debían garantizar a la sociedad. La tendencia neoliberal resucitaba el viejo liberalismo económico y manchesteriano: mercancías y servicios deben ser producidos y distribuidos por el mercado con la

${ }^{21}$ Friedrich A. Hayek, Camino de servidumbre (San José: Universidad Autónoma de Centro América, 1986), 89. 
mínima intervención estatal, satisfaciendo de ese modo las necesidades y deseos de la gran mayoría de la población (la consecuencia era que el Estado de bienestar debía ser desmantelado y la mayor parte de los servicios públicos, privatizados). La corriente neoconservadora, aunque aceptaba el mercado como productor de riqueza, pensaba también que erosionaba la moral y el orden social: en realidad defendía más la propiedad privada que el libre mercado y, sobre todo, hacía énfasis en la benéfica influencia de la tradición, la jerarquía y la moral que sólo instituciones como la familia patriarcal, un Estado fuerte y la religión anglicana pueden garantizarla; para ellos, el enemigo era el relativismo moral y la permisividad social.

Obviamente esta dualidad ideológica dentro de la derecha británica tenía que plantear problemas a la hora de regular la educación. La educación puede ser considerada de dos formas diferentes: como un servicio del Estado, un servicio de los que el propio Adam Smith consideraba que nunca deberían quedar en manos de un individuo o un pequeño grupo, sino de las «instituciones públicas», o, y esta era la posición neoliberal, la educación debía ser un servicio completamente privado. Como la educación pública, fruto de una larga evolución de los siglos XIX y XX, no podía ser totalmente suprimida, el neoliberalismo inglés apostó por la máxima privatización de la educación, siendo la libertad de elección de centro el elemento central de la nueva política. El neoconservadurismo consideró, sin embargo, que la educación era demasiado importante para dejarla en manos del mercado, por lo que reclamaba un Estado lo suficientemente capaz de restaurar e imponer los viejos valores tradicionales, transmitidos a través de las escuelas.

Esta situación produjo importantes tensiones en el seno del thatcherismo, sólo resuelto por el genio pragmático inglés que concilió ambas tendencias en la ley de 1988 gracias a un complicado mecanismo de concesiones recíprocas. En este juego de cesiones mutuas, los neoliberales aceptaron en la nueva ley un currículo nacional, algo insólito en el Reino Unido, y con él la vuelta de los valores tradicionales: el currículo nacional, obligatorio para todas las escuelas, hará énfasis en las materias académicas tradicionales, su observancia se comprobará con las pruebas de evaluación externas, preparadas por las autoridades centrales- lo que implicaba en realidad una centralización como Inglaterra no había conocido nunca y un fortalecimiento del ministerio inglés desconocido desde 1944-, si bien los neoliberales introdujeron en el currículo las ideas de libre empresa, competitividad y eficiencia. A cambio de un currículo prescrito y nacional, los neo- 
conservadores rebajaron sus pretensiones de un Estado fuerte, admitieron el adelgazamiento relativo del Estado y aceptaron una libertad de elección de centro suavizada en sus pretensiones. Como se ha indicado, la ley de 1988 esconde una gran paradoja: es una ley que aumenta notablemente el papel de las fuerzas del libre mercado en la educación al mismo tiempo que regula detalladamente el incremento de las atribuciones del ministerio de Educación inglés e impone, por primera vez en la historia de Gran Bretaña, un currículo prescriptivo de carácter nacional. ${ }^{22}$ El thatcherismo terminó siendo una ideología ecléctica que combinaba la libertad de elección de centro docente - aportación neoliberal- con el currículo prescriptivo de carácter nacional —aportación neoconservadora-.

Era precisa esta referencia al thatcherismo porque esa tensión interna se reprodujo en la refundación del partido conservador, en la creación del nuevo Partido Popular (PP) que surgió bajo la dirección de Aznar en 1990, tensión que se manifestó tanto en el primer gobierno popular (1996-2004) como en el segundo (2011-2015). De una parte, el ala neoliberal del partido se mostró partidaria de la libertad de elección de centro y, como una consecuencia obligada, optó decididamente por la libre competencia entre los centros docentes que, para atraer más alumnos, mejorarían la calidad de la educación prestada, de modo que sin coste económico alguno para el Tesoro público se produciría la mejora de la educación gracias a una mayor privatización de la educación. Pero el neoliberalismo tenía que convivir con la otra tendencia, partidaria de la vuelta a los valores básicos de la tradición conservadora: los nuevos conservadores españoles, alarmados por lo que consideraban una grave crisis del mundo occidental, pensaban que era necesario reforzar las viejas virtudes de la familia patriarcal, de los valores nacionales que produjeron las glorias pasadas y de las creencias religiosas establecidas mayoritariamente en el país, así como los de la pedagogía tradicional, esto es, la cultura del esfuerzo y de la disciplina, la autoridad del profesor y la presencia de la religión en el aula como disciplina fundamental.

Cuando el PP ganó las elecciones generales en 2011 por una aplastante mayoría, las dos almas del partido entraron en pugna. La compleja elaboración de la LOMCE a lo largo del primer bienio del gobierno popular,

${ }^{22}$ Geoff Whitty, «The new right and the national curriculum: state control or market forces?», en The Education Reform Act 1988, eds. Michael Flude y Merril Hammer (London: The Falmer Press, 1990). 
2011-2013, ha sido una prueba evidente de esta tensión: hubo un primer borrador, fuertemente neoliberal y economicista, que levantó una gran polvareda entre los neoconservadores del partido: no vieron en ese borrador reflejadas sus demandas. Se produjeron entonces diversas modificaciones en sucesivos borradores, modificaciones que, huelga decirlo, no obedecían a las demandas de la comunidad educativa o de las demás fuerzas sociales y políticas - lo que hubiera dado lugar, al menos, a una ley de relativo consenso-, sino a las presiones internas del sector neoconservador del PP. Ahora bien, aunque hubo importantes concesiones como respuesta a esas presiones, una lectura del texto aprobado en el Congreso de los Diputados a finales de 2013 arroja, en mi opinión, un fuerte acento en la libertad de elección de centro y un claro ascenso de la tendencia privatizadora, lo que indica que, en el difícil equilibrio de las dos almas del PP, predominó más el neoliberalismo.

\section{CONSENSO, DISENSO Y PACTO EN EDUCACIÓN}

No voy a analizar ahora la LOMCE, pero sí la situación que ha creado esta ley, aprobada prácticamente con la oposición de todo el arco parlamentario a excepción del voto del PP. Nada de esto es nuevo. Lo nuevo es que la LOMCE, apoyada en la prepotencia de la mayoría absoluta, haya provocado algo verdaderamente inédito en el Congreso de los Diputados: los partidos de la oposición, salvo obviamente el que sustentaba al gobierno y un pequeño grupo parlamentario, firmaron un pacto en el que se comprometieron a derogar la LOMCE cuando se produjera una nueva alternancia de gobierno. Asistimos así a una fuerte aceleración de una espiral ya existente, que desembocará en una mayor inestabilidad del sistema educativo, alimentará la nociva dialéctica de reformas y contrarreformas, causará una enorme desmoralización en el profesorado y, lo que es más penoso, impedirá la debida atención a los problemas más urgentes de la educación, algo que debería concitar los esfuerzos de todos los gobiernos, sea cual fuere su color político. Esta situación explica quizá que sean ya muchas las voces que reclaman un nuevo consenso en educación. ¿Quiere esto decir que el pacto constitucional del artículo 27 está superado? No exactamente.

En primer lugar, el consenso no supone ausencia de conflicto, ni el fin de las ideologías ni el eclipse de políticas diferentes, pero tampoco se debe ignorar que estas diferencias no deben ocultar la existencia de espacios comu- 
nes que pueden permitir acuerdos y que «es igualmente importante resaltar aquello en que los miembros de la sociedad están de acuerdo"; 23 por tanto, hay que tener en cuenta no sólo los valores e intereses que dividen, también son importantes los que unen a la sociedad. ¿Cuáles son esos aspectos que configuran un espacio común sobre el que es posible converger, al menos para formular políticas de mejora de la educación? No descubrimos nada nuevo si afirmamos que hay demandas en nuestra sociedad que apuntan directamente hacia determinados problemas que es necesario resolver: la formación y selección del profesorado, el fracaso y el abandono escolares, la renovación profunda del currículo básico, la reforma profunda de la formación profesional, el necesario incremento de la inversión pública y algunos puntos concretos más. Estos son aspectos importantes de la educación que podrían mejorar si se llegara a acuerdos sobre las políticas a implementar. Asimismo, es necesario mejorar las relaciones entre las dos redes de educación que en nuestro país se financian con fondos públicos - las escuelas públicas y las escuelas concertadas-. Consideradas como antagónicas, hora es ya que de comiencen a funcionar como complementarias. Para ello es preciso que, como ocurre en la mayoría de los países de la Unión Europea, la escuela pública sea de verdad el eje vertebrador de la educación, que sea patrimonio común de todos y que la escuela concertada coadyuve a la función pública que la educación cumple, recibiendo para ello las ayudas necesarias.

¿Por qué no se llegan a formalizar estos acuerdos, a pesar de que esté calando en la sociedad española la idea de que para mejorar la educación es más necesario el acuerdo que el desacuerdo? Posiblemente porque en las fuerzas sociales y políticas persiste aún el temor a que un acuerdo en educación signifique una deslealtad a los propios principios (cuando no a los intereses). Al menos esta es la lección que nos ofrece la pequeña historia de los pactos intentados en los últimos lustros.

El 17 de septiembre de 1997 se firmó en Madrid la Declaración conjunta en favor de la educación por dieciocho organizaciones del mundo de la educación. Cuando se discutían las posibilidades de un pacto entre estas organizaciones las partes intervinientes buscaban, por un lado, el consenso, pero, por otro, querían salvaguardar ante sus bases los principios, es decir,

${ }^{23}$ Denis Lawton, Education and Politics in the 1990s. Conflict or Consensus? (London: The Falmer Press, 1994), 110. 
se debatían entre el consenso y el disenso. De esta forma, aunque el documento fue un gran paso en esa búsqueda de un espacio común y un precedente importante, se evidenciaron también las dificultades para lograrlo. Ello explica que la Declaración fuera acompañada de diversos anexos en los que las más importantes organizaciones y asociaciones mostraban los aspectos sobre los cuales no se había llegado a acuerdo alguno, cuestión ésta, por lo demás, recogida incluso en el propio texto: «La firma conjunta de esta Declaración no supone compartir los mismos puntos de vista en los problemas educativos como consta en los escritos que presentan las organizaciones en relación con la firma de la presente Declaración» ${ }^{24}$. Era reconocer la presencia del disenso y la dificultad de conciliarlo con el consenso.

Pocos años después, en marzo de 2005, análoga dificultad se presentó en el segundo intento por alcanzar un acuerdo social sobre la educación, fruto de una iniciativa surgida en el seno del Consejo Escolar del Estado. A pesar del extraordinario esfuerzo realizado por Comisiones Obreras (CC OO), la Federación de Religiosos de la Enseñanza (FERE), y la Federación de Trabajadores de la Enseñanza (FETE), a la hora de firmar el Pacto social por la educación, las dos organizaciones nacionales de padres y madres de alumnos rechazaron firmarlo. Como he indicado en otro lugar, las organizaciones de padres

sucumbieron frente a fuerzas internas que se mostraron partidarias de una radical fidelidad a los principios. [...] El consenso no es posible cuando los valores básicos de unos y otros se mantienen en su irreductible pureza, creándose una falsa mecánica en la que unos aparecen como los depositarios de las esencias y otros como los pragmáticos claudicadores. ${ }^{25}$

Finalmente, la sombra del disenso apareció de nuevo cuando por primera vez en nuestra historia un ministro planteó en 2009 , como voluntad del Gobierno, la idea de alcanzar un pacto en educación con la comunidad escolar, los agentes sociales, los partidos políticos y las comunidades autónomas. El ministro Gabilondo mantuvo a lo largo de un año múltiples y numerosos encuentros con todos los actores invitados al pacto, presentando

\footnotetext{
${ }^{24}$ Declaración conjunta en favor de la educación (Madrid: Fundación Encuentro, 1997), 6.

${ }_{25}$ Manuel de Puelles Benítez, "¿Por qué no fue posible el pacto educativo?», Organización y Gestión Educativa, 14 (6), (2006): 19.
} 
en abril de 2010 lo que denominó Propuestas para un pacto social y político de la educación. Como es sabido, el PP rehusó la firma alegando que el Gobierno quería blindar un modelo que había fracasado por completo, lo que, a contrario sensu, implicaba apostar por otro modelo, es decir, otra visión de la educación, la suya.

¿Qué nos enseña esta pequeña historia? En primer lugar, que el pacto, de conseguirse, no supondrá el fin de los conflictos, ni las ideologías enfrentadas dejarán de existir, ni las políticas educativas serán en adelante planas y uniformes. El disenso permanecerá porque constituye la esencia de la vida democrática, porque los diferentes gobiernos, aun consensuando las reformas y las leyes que las impulsan, aplicarán sus políticas haciendo más énfasis en unos valores que en otros. Por razones bien conocidas, la izquierda política tenderá a acentuar las exigencias constitucionales derivadas del principio de igualdad, centradas sobre todo en el acceso de todos a la educación, mientras que la derecha política tenderá a acentuar los derechos constitucionales derivados del principio de libertad, centrados sobre todo en la libertad de elección de centro, pero ambas políticas seguirán siendo legítimas si, como ha afirmado reiteradamente el Tribunal Constitucional, se respeta el contenido básico de los derechos y libertades consensuados en el artículo 27 de la Constitución cuyo apartado primero dice: «Todos tienen el derecho a la educación. Se reconoce la libertad de enseñanza». ${ }^{26}$ Como se ha señalado, a mi parecer con acierto, el artículo 27.1

condensa la oposición entre dos derechos contrapuestos y complementarios, el de igualdad y el de libertad. Dos derechos en conflicto que figuran en el texto constitucional, uno junto a otro, tras ser propuestos, respectivamente, por la izquierda y la derecha del espectro político. ${ }^{27}$

En buena parte, respetar el contenido básico del derecho a la educación y de la libertad de enseñanza no supone sólo buscar ese espacio común a que antes nos referíamos, sino también superar el pensamiento dicotómico que nos presenta el problema como una inevitable opción por los derechos de libertad o por los derechos de igualdad. En parte porque el principio de

\footnotetext{
${ }^{26}$ Constitución española (Madrid: Boletín Oficial del Estado, 1979): 30.

27 Antonio Viñao, «El desmantelamiento del derecho a la educación: discursos y estrategias neoconservadoras», Áreas. Revista Internacional de Ciencias Sociales, 31 (2012): 98. La cursiva es mía.
} 
libertad admite varias interpretaciones, es decir, no se limita al derecho a crear, dirigir y elegir centros docentes: ¿cómo en una sociedad liberal el sistema escolar no va a educar para la libertad, no va a formar personas para el ejercicio responsable de las libertades civiles? ¿Cómo rechazar que hay que formar a los niños y a las niñas en el respeto a la libertad de conciencia, en el derecho a la libre expresión de las ideas, en el ejercicio de la libertad de reunión o de asociación? Incluso la tan debatida libertad de elección de centro, ¿cómo no aceptar que hay que promover las condiciones para que esa libertad sea igual para todos, que todos puedan verdaderamente elegir el centro que desean? En parte también porque ¿cómo en una sociedad democrática no se va a educar en libertad, preparando a las personas para la participación formando ciudadanos? ¿Cómo no instrumentar políticas dirigidas a hacer de la escuela una comunidad real, promoviendo el uso de metodologías activas en el proceso de enseñanza y aprendizaje, produciendo cambios sustanciales en la relación alumnos-profesor, fomentando la participación en la vida diaria de la escuela? Sólo así habrá una escuela en la que los niños y los adolescentes, mediante la práctica democrática en el propio centro docente, aprendan y se preparen para ser ciudadanos. Asimismo, ¿cómo en una sociedad liberal y democrática no se va a extender al máximo el derecho de todos a la educación, un derecho básico consagrado en la Declaración Universal de las Naciones Unidas sobre Derechos Humanos? No obstante, y en todo caso, bastaría que las fuerzas políticas, defendiendo su interpretación de los derechos y libertades, respetaran el contenido básico indicado, aunque después hicieran un aplicación más o menos extensiva, más o menos restrictivas de esos derechos y libertades.

En segundo lugar, en mi opinión, es necesario completar el artículo 27 de la Constitución porque la tendencia creciente a favor de la reforma constitucional no puede limitarse a la reordenación territorial del Estado, con ser esta una cuestión muy importante, sino también a la reordenación de derechos fundamentales como son la educación y la sanidad, tan necesitados hoy de una adecuada protección constitucional. Respecto de la educación, aunque el consenso del artículo 27, como ya hemos indicado, tuvo suficiente virtualidad para superar errores históricos del pasado, su aplicación, sin embargo, no ha podido evitar que las políticas de los sucesivos gobiernos hayan incurrido en una continua inestabilidad legislativa con la consiguiente dialéctica de reformas y contrarreformas. Es necesario, pues, un nuevo consenso constitucional en educación que refuerce las garantías de los de- 
rechos básicos que se derivan de los principios de libertad de enseñanza y de igualdad ante la educación, reconocidos y colocado al mismo nivel por el artículo 27; es necesario consensuar un marco garantista de carácter constitucional que impida la supremacía de los derechos de libertad sobre los derechos de igualdad, y viceversa, así como proteger estos derechos frente a escandalosas políticas de recortes.

En tercer lugar, el camino de la reforma constitucional será probablemente largo y dificultoso, pero mientras ese proceso se realice es preciso abrir otras vías de diálogo y de consenso. Se trata, pues, de explorar nuevos procesos de entendimiento, abrir un camino hacia objetivos menos ambiciosos, un camino que facilite el logro de un acuerdo de mínimos capaz de preparar y facilitar más tarde la reforma constitucional. ¿Cómo lograr ese acuerdo? ¿Cómo evitar mientras tanto el círculo vicioso de leyes y más leyes de educación? ¿Cómo conseguir una política de reformas dirigida a mejorar la educación, esto es, sostenidas a medio y largo plazo? Posiblemente haya que caminar por la senda de los acuerdos mínimos, una senda que pueda conducirnos

hacia el logro de un triple acuerdo: a) sobre el diagnóstico del sistema educativo, dejando fuera los análisis interesados, utilizados muchas veces como arma arrojadiza contra el adversario político; b) sobre los problemas más importantes que arroje ese diagnóstico -muchos de ellos están ya en el imaginario actual del mundo educativo-; c) sobre las políticas encaminadas a resolverlos para que puedan mantenerse tras la alternancia democrática — la experiencia de estos años muestra que, para ser fecundas, tienen que ser sostenibles a corto y medio plazo-. Para estos acuerdos no se necesitan más leyes..$^{28}$

Acordar un diagnóstico es un paso previo y sustancial: hay que arrojar al olvido los análisis que tantas veces se han utilizado como justificación partidista de las reformas propias. El segundo paso sería consecuencia del primero: un acuerdo sobre los problemas que revele el diagnóstico (muchos de ellos, como hemos indicado, están ya en el imaginario actual del mundo educativo). Finalmente, un acuerdo sobre las políticas que puedan resolver

28 «Aportaciones del Colectivo Lorenzo Luzuriaga al debate educativo en un año electoral. Marzo 2015». http://www.colectivolorenzoluzuriaga.com/novedades (consultado el 14-5-2015). 
esos problemas y que puedan sostenerse tras la alternancia democrática, desterrando ese vicio adánico de la política española de destruir los avances conseguidos por el gobierno anterior para volver a empezar de nuevo.

Hay, pues, que romper el nudo gordiano, hay que desterrar el maleficio que el mito de Sísifo despliega sobre nosotros: somos como Sísifo que, castigado por sus engaños a los dioses, tuvo que cargar sobre sus espaldas una gran roca hasta la cima de una montaña para, desde allí, descargarla y... volver a empezar de nuevo. Como Sísifo, la alternancia democrática de fuerzas contrarias al actual gobierno, cuando ésta se produzca, se verá obligada, una vez más, a derogar una ley de educación y preparar otra. Y Sísifo volverá, otra vez, a redoblar sus inútiles esfuerzos. La verdad, creo que este país no se merece esto.

\section{Nota sobre el autor:}

Manuel de Puelles, catedrático de Política de la Educación en la UNED, ha desempeñado diferentes cargos en el Ministerio de Educación y ha sido asesor de la Organización de Estados Iberoamericanos para la Educación, la Ciencia y la Cultura (OEI), experto del Consejo de Europa y vicepresidente del Consejo Escolar del Estado. Ha sido además director de la Revista Iberoamericana de Educación (19941999) y consejero de la Revista de Educación del Ministerio de Educación, entre otras actividades. Fue también impulsor y director del proyecto de investigación sobre los manuales escolares españoles de la época contemporánea que dio lugar a la creación del Centro de Investigación MANES, que lleva ya más de veinte años de existencia. Entre su amplia producción destacan libros como Educación e ideología en la España contemporánea (Barcelona: Labor, 1980 - reeditado por Técnos); Estado y educación en la España liberal (1809-1857): un sistema educativo nacional frustrado (Barcelona: Pomares, 2004) y Modernidad, republicanismo y democracia: una historia de la educación en España, 1898-2008 (Tirant lo Blanch, 2009). 